\title{
North-south asynchrony and long-term variations of sunspot latitudes
}

\author{
Nadezhda V. Zolotova $†$ and D. I. Ponyavin \\ Institute of Physics, Saint-Petersburg State University, Russia \\ email: ned@geo.phys.spbu.ru
}

\begin{abstract}
The long-term records of sunspot area available separately for Northern and Southern Hemispheres have been investigated by means of cross-recurrence technique. Phase component of the north-south asymmetry was extracted. This measure demonstrates long-period systematic variations with the sign change of hemispheric leading in 1930s and 1960s. Moreover phase north-south asynchrony anticorrelates with the so called magnetic equator, which was defined as difference of the mean sunspot latitudes between two hemispheres. Relationships of the phase north-south asynchrony, magnetic equator and butterfly diagrams are presented and discussed.
\end{abstract}

Keywords. Sun: activity - sunspots - magnetic fields

\section{Introduction}

Variations of sunspot latitudes from 1853 to 1996 were analysed by Pulkkinen et al. (1999). They have introduced the so called magnetic equator as the sum of mean $\langle\lambda(N)\rangle_{n}+$ $\langle\lambda(S)\rangle_{n}$ (the southern component is negative, $\mathrm{n}$ is the time epoch). Thus the magnetic equator traces a skewness in distribution of sunspot latitudes with respect to equator. A systematic variation with a period of about 90 years was revealed (Pulkkinen et al. 1999).

In this work we compare long-term behavior of the magnetic equator with phase north-south hemispheric asynchrony. In order to extract the last one we used the crossrecurrence plots (http://www.agnld.uni-potsdam.de/ marwan/toolbox/):

$$
C R_{i, j}=\Theta\left(\varepsilon_{i}-\left\|\mathbf{x}_{i}-\mathbf{y}_{j}\right\|\right), \quad i, j=1, \ldots, N,
$$

where $N$ is the number of considered states $\mathbf{x}, \mathbf{y},\|\cdot\|$ is a norm, and $\Theta$ is the Heaviside function (Marwan et al. 2007).

\section{Results and conclusion}

Extracted from cross-recurrence plot the Line of Synchronization (LOS) traces time delays between considered time series. LOS for the smoothed monthly northern and southern hemispheric sunspot area is presented in figure 1(a).

The change of the LOS sign indicates the change of the leading role of the hemispheres (LOS $>0$ - north leading, LOS $<0$ - south leading). It is seen that before the end of cycle 16 the north dominates in leading, after that till the minimum just before the cycle 20 , the southern hemisphere leads, and than up to the present, the northern hemisphere leads again.

On the figure 1(b) the smoothed separate mean sunspot latitudes of northern and southern hemisphere is shown. It reveals that up to the end of the solar cycle 16 the north

$\dagger$ Present address: Institute of Physics, Ulyanovskaya ul., 1, Petrodvorets, 198504, Russia. 


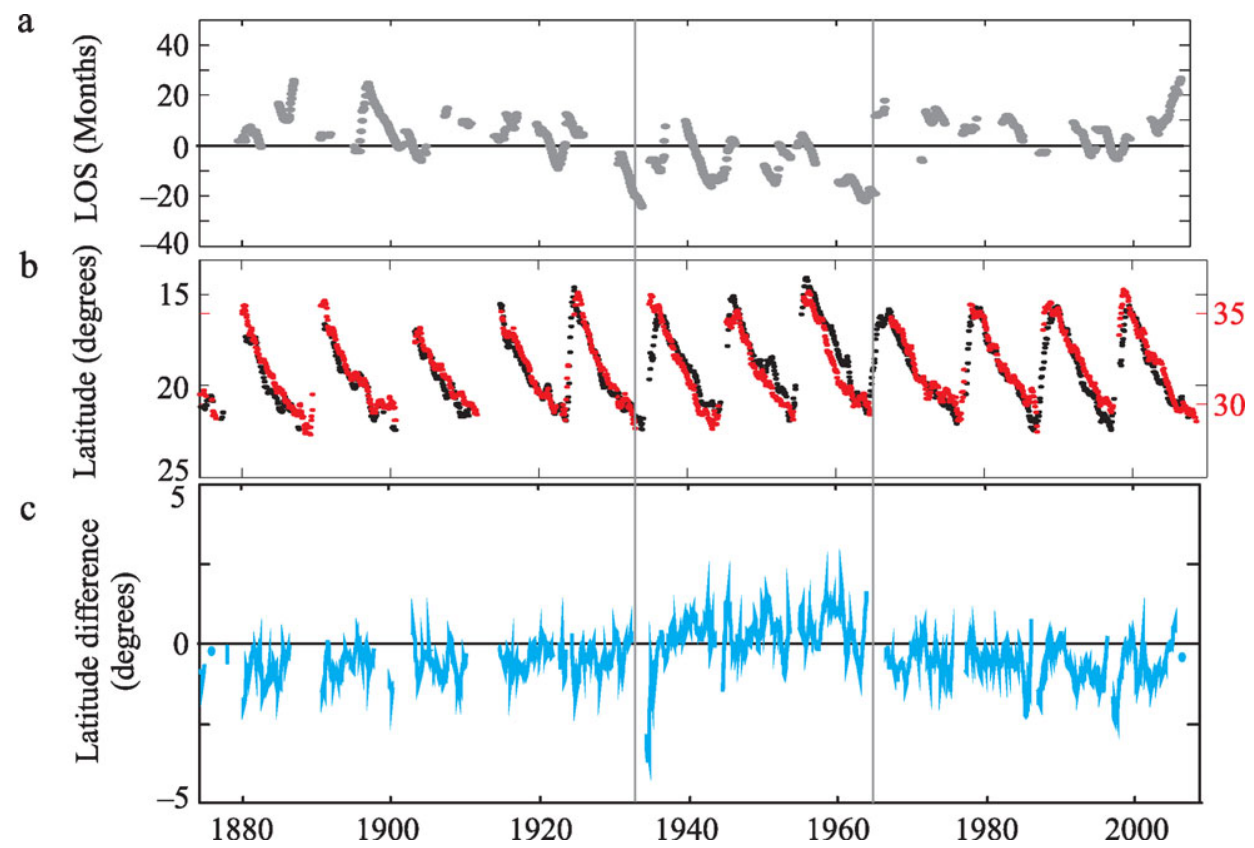

Figure 1. (a) Line of Synchronization for hemispheric sunspot area; (b) Smoothed mean sunspot latitudes for the north (black) and south (red); (c) Their difference - the magnetic equator. similar to results of Pulkkinen et al. (1999).

is leading in time, but with preference of sunspots to emerge at the higher latitudes in the south. After this epoch and until the beginning of cycle 20, the situation is reversed to opposite. After cycle 20 it is restored back to a situation as appeared before cycle 16 .

The differences between these mean sunspot latitudes (figure $1 \mathrm{c}$ ) clearly reproduce variations of the magnetic equator. Moreover, the results coincide with the weighted magnetic equator derived by Pulkkinen et al. (1999). Analyzing time series extended to 1853 (Carrington's and Spörer's data) they found additional zero crossing just before the cycle 12. They concluded that there are regular magnetic equator variations close to the Gleissberg cycle.

In our work we observed that intervals between zero crossings are not equal to each other, probably suggesting process of stochastic or chaotic nature. Moreover it is interesting to note that asymmetry at the end of prolonged cycle 23 is unusually high. Thus we expect that some more dramatic change of the solar dynamics may occur in the nearest future.

\section{Acknowledgements}

We used the RGO USAF/NOAA data of sunspot area and their latitudes http://solarscience.msfc.nasa.gov/greenwch.shtml.

This research has been supported by the INTAS Fellowship Grant for Young Scientists Ref. No 06-1000014-6022.

\section{References}

Marwan, N., Romano, M. C., Thiel, M., \& Kurths, J. 2007, Phys. Reports 438, 237

Pulkkinen, P. J., Brooke, J., Pelt, J., \& Tuominen, I. 1999, A\&A A 341, L43 